\title{
Analysis of Transport Properties of the Randomly Moving Electrons in Metals
}

\author{
Vilius PALENSKIS ${ }^{1}$, Evaras ŽITKEVIČIUS ${ }^{2}$ * \\ ${ }^{1}$ Physics Faculty, Vilnius University, Sauletekio al. 9, LT-10222 Vilnius Lithuania \\ ${ }^{2}$ Department of Electronic Systems, Vilnius Gediminas Technical University, Naugarduko 41, LT-03227 Vilnius, Lithuania \\ crossref http://dx.doi.org/10.5755/j01.ms.26.2.21730
}

Received 28 September 2018; accepted 20 February 2019

\begin{abstract}
In this critical analysis on the base of randomly moving (RM) electrons, presented the resistivity dependence on temperature for elemental metals both above and below the Debye's temperatures. There also are presented the general relationships for estimation of the average diffusion coefficient, the average velocity, mean free path and average relaxation time of RM electrons on the Fermi surface at mentioned temperature range. It is shown that the scattering of RM electrons mainly is due to electronic defects associated with distortion of the periodic potential distribution in the periodic lattice, and accounting the exchange of the thermal energies between phonon and RM electron. The calculation results of resistivity dependence on temperature in the temperature range from $1 \mathrm{~K}$ to $900 \mathrm{~K}$ are demonstrated for Au and $\mathrm{W}$ and compared with the experimental data. There also is presented the simple method for determination of the basic kinetic characteristic dependences on temperature only from the resistivity dependence on temperature. It is at first time determined for $\mathrm{Au}$ and $\mathrm{W}$ the temperature dependences of the mean free path, average diffusion coefficient, average relaxation time of RM electrons from $1 \mathrm{~K}$ to $900 \mathrm{~K}$.

Keywords: resistivity, conductivity, electronic defects, electron mean free path, average electron relaxation time.
\end{abstract}

\section{INTRODUCTION}

The foundations of the electron theory of metals were laid on the preposition of free electrons, which are assumed to be of the same order of valence electrons per unit volume, which contradicts to the Fermi-Dirac statistics for electrons. In order to obtain the correct order of the magnitude for conductivity and correct temperature variation of the mean free path of electrons at room temperature range, it was assumed that electron mean path is caused by thermal vibrations of lattice [1-8]. It is well known that electrons obey the Pauli principle and that electrons are described by the Fermi-Dirac statistics. The latter statistics let to explain the experimental results of the electron heat capacity of metals: why metals and insulators have around the same heat capacity. The resolution of this paradox is one of the greatest successes of the Sommerfeld's model [3].

According to Fermi-Dirac statistics and Pauli principle description of the electrical conductivity of the metals including all valence electrons is unacceptable $[9,10]$, because randomly can move only a small part of electrons which energy is close to the Fermi level energy, and that electrons which energy is well below the Fermi level energy cannot change their energy, because all neighbor energy levels are occupied. Concerning that the Sommerfeld's model is based on the spherical Fermi surface, there also are uncertainties in determination of both the density-of-states (DOS) in conduction band and the Fermi energy, because the Fermi surfaces for many of metals are not spherical [11]. The determination of the effective density $n_{\text {eff }}$ of the randomly moving (RM) electrons in elemental metals eliminates the mentioned uncertainties $[9,10]$.
This critical analysis mainly is addressed to application of the effective density of RM electrons for description of the basic electron transport characteristics of metals, and to their dependence on temperature. It is that demonstrated for $\mathrm{Au}$ and $\mathrm{W}$ in the temperature range from $1 \mathrm{~K}$ to $900 \mathrm{~K}$. The mentioned problems are very important for solid state physics.

\section{RESULTS AND DISCUSSION}

\subsection{Stochastic description of $\mathrm{RM}$ electrons in metals}

According to the Fermi-Dirac statistics and Pauli exclusion principle, the probability of occupation of energy states in conduction band by electrons at thermal equilibrium is described by Fermi distribution function $f(E)$ :

$$
f(E)=1 /[1+\exp (E-\eta) / k T]
$$

where $E$ is the electron energy; $\eta$ is the chemical potential; $k$ is the Boltzmann's constant, and $T$ is the absolute temperature. Considering that the difference between the chemical potential and Fermi level energy $E_{\mathrm{F}}$ is only about $0.01 \%$ for room temperature [3], further for calculation we use the Fermi distribution function in the following form:

$$
f(E)=1 /\left[1+\exp \left(E-E_{\mathrm{F}}\right) / k T\right] \text {. }
$$

Thus, the total density of the free valence electrons $n$ in the conduction band is described by density-of-states (DOS) $g(E)$ of the unit volume as

$$
n=\int_{0}^{\infty} g(E) f(E) \mathrm{d} E .
$$

\footnotetext{
* Corresponding author. Tel.: +370-60724710.

E-mail address: evaras.zitkevicius@vgtu.lt (E. Zitkevicius)
} 
The electrical conductivity depends not only on the DOS $g(E)$ in conduction band and Fermi distribution function $f(E)$, but it also depends on the probability $f_{1}(E)=1-f(E)$ that any electron with the definite energy $E$ at a given temperature $T$ can be thermally excited or can change his energy under influence of the external fields. The effective density of electrons $n_{\text {eff }}$ which take part in random motion and in conductivity depends on the probability:

$h(E)=f(E)[1-f(E)]$.

Therefore, the effective density of randomly moving electrons $n_{\text {eff }}$ is described as $[9,10]$

$n_{\text {eff }}=\int_{0}^{\infty} g(E) f(E)[1-f(E)] \mathrm{d} E$

$=k T \int_{0}^{\infty} g(E)(-\partial f(E) / \partial E) \mathrm{d} E$.

From Eq. 5 it follows that the term $(-\partial f(E) / \partial E)$ is the probability density function $p(E)$ that electron having the energy $E$ can randomly move and also can change its energy due to thermal and electrical influences:

$$
p(E)=(-\partial f(E) / \partial E)=h(E) / k T .
$$

The function $p(E)$ meets the requirements of the probability theory, and also accounts the Pauli exclusion principle.

The Eq. 5 is valid in all cases: for non-degenerate and degenerate electron gas with one type of charge carriers. For materials with non-degenerate electron gas the probability $[1-f(E)] \approx 1$ because $f(E)<<1$, and, therefore, all electrons in conduction band $n$ take part in random motion, and their density can be described as

$n_{\mathrm{eff}}=n=\int_{0}^{\infty} g(E) f(E) \mathrm{d} E$.

This is the case when the classical statistics is applicable. In the case of highly degenerate electron gas, and considering that probability density function $(-\partial f(E) / \partial E)$ has a sharp maximum at $E=E_{\mathrm{F}}$, the Eq. 5 can be presented in the following form:

$n_{\text {eff }}=g\left(E_{\mathrm{F}}\right) k T<<n$,

where $g\left(E_{\mathrm{F}}\right)=g(E)$ at $E=E_{\mathrm{F}}$. The DOS at Fermi energy $g\left(E_{\mathrm{F}}\right)$ can be obtained from the measurements of the electron heat capacity of elemental metals [13].

Illustration of the effective density of RM electrons at room temperature and $E_{\mathrm{F}}=2 \mathrm{eV}$ for elemental metals and normal state superconductors with the composite DOS is presented in Fig. 1. It must be pointed that the effective density of RM electrons is completely described by DOS at Fermi surface, but not by the total density of free valence electrons.

\subsection{Electrical conductivity of metals}

The electrical conductivity $\sigma$ of the homogeneous materials with one type of randomly moving carriers (electrons or holes) can be described by such general expression [12]: $\sigma=\frac{2 q^{2}}{3 m_{\sigma} k T} \int_{0}^{\infty} \tau(E) g(E) f(E)[1-f(E)] E \mathrm{~d} E$,

where $\tau(E)$ is the relaxation time of electrons with kinetic energy $E$ in conduction band, $m_{\sigma}$ is the conductivity effective mass.

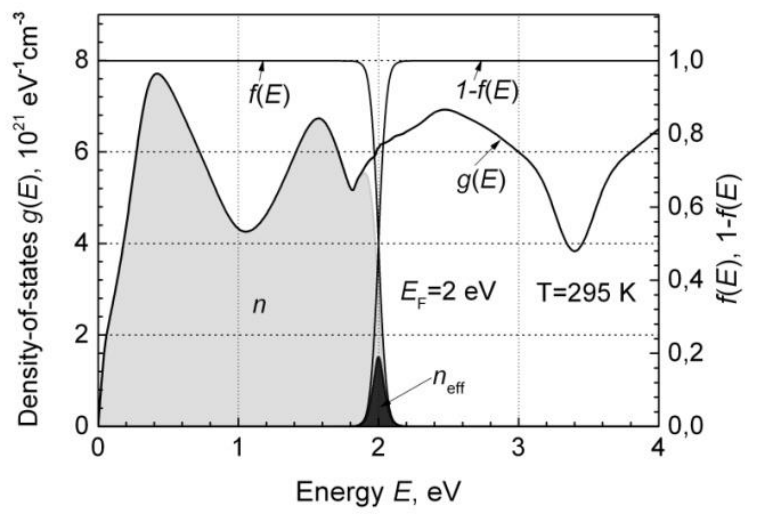

Fig. 1. Illustration of the effective density of RM electrons at room temperature $(295 \mathrm{~K})$, and $E_{\mathrm{F}}=2 \mathrm{eV}$ for metals and normal state superconductors with the composite DOS. The light grey area represents the total density of free electrons $n$ in conduction band, and the black area represents the effective density $n_{\text {eff }}$ of RM electrons

This expression after simple transfer can be presented as [14]:

$\sigma=\frac{q^{2} D}{k T} \int_{0}^{\infty} g(E) f(E)(1-f(E)) d E$

or as

$\sigma=\frac{q^{2} D}{k T} n_{\mathrm{eff}}$

where $D=(1 / 3)<v^{2}>\cdot<\tau>$ is the diffusion coefficient of $\mathrm{RM}$ electrons, here $\mathrm{v}$ and $\tau$, respectively is the velocity and relaxation time of RM electrons. The conductivity also can be described as [14]:

$\sigma=q^{2} D\left(\frac{\partial n}{\partial \eta}\right)_{T}$

where $\eta$ is the chemical potential. There all Eq. $9-$ Eq. 12 are equivalent, because they can be derived one from other. Moreover, they are valid in both cases: for homogeneous material with non-degenerate and degenerate electron gas. From Eq. 11 follows such general description of the drift mobility of RM electrons:

$\mu_{\mathrm{drift}}=\frac{q D}{k T}=\frac{q<v^{2}>\cdot<\tau>}{3 k T}=\frac{q<\tau>}{m_{\sigma}} \cdot \frac{<E>}{(3 / 2) k T}$,

where $\langle E\rangle=m_{\sigma} v^{2} / 2$ is the average kinetic energy of RM electrons. The Eq. 13 also is valid for all homogeneous materials with a single type of charge carriers at any their degeneracy degree [15]. For metals the Eq. 11 can be rewritten as 
$\sigma=\frac{1}{\rho}=q^{2} g\left(E_{\mathrm{F}}\right) D=\frac{1}{3} q^{2} g\left(E_{\mathrm{F}}\right) \nu_{\mathrm{F}}^{2} \tau_{\mathrm{F}}$.

The diffusion coefficient $D$ of RM electrons for various metals can be estimated from Eq. 14. The data of the experimental values of the electrical conductivity and the electronic heat capacity for different metals at $T=295 \mathrm{~K}$ is taken from [13].

From Eq. 14 it follows that diffusion coefficient of RM electrons is related minimum with the five transport parameters of RM electrons:

$D=\frac{\sigma}{q^{2} g\left(E_{\mathrm{F}}\right)}=\frac{1}{q^{2} g\left(E_{\mathrm{F}}\right) \rho}=\frac{1}{3} v_{\mathrm{F}}^{2} \tau_{\mathrm{F}}=\frac{1}{3} l_{\mathrm{F}} v_{\mathrm{F}}$,

where $l_{\mathrm{F}}=v_{\mathrm{F}} \tau_{\mathrm{F}}$ is the mean free path of the electrons on the Fermi surface. What parameters mostly cause the spread of the resistivity and diffusion coefficient of RM electrons for metals at $E=E_{\mathrm{F}}$ ?

\subsection{Scattering of $R M$ electrons in metals at temperatures above the Debye's temperature}

The very important parameter characterizing the scattering mechanism of RM charge carriers is their mean free path. According to the quantum mechanics, the free electrons can freely move in ideal periodic lattice of the metal crystal without any scattering by ions $[3,7]$. Therefore, the resistivity of the ideal crystal with periodic lattice having periodic distribution of the potential energy must be equal to zero. Scattering of electron can be only in the spots where there are distortions of the periodicity of the potential energy of the ideal lattice structure. The resistivity of metals is caused due to scattering of free electrons by impurities, vacancies, interstitial atoms, dislocations, boundaries of grains, and by surface. An usual explanation considering that all free valence electrons $n$ are scattered due to increasing the amplitude of thermal vibration of atoms in lattice, and that causes the increase of electron-phonon scattering cross-section $\sigma_{\mathrm{el}-\mathrm{ph}}[1-8]$ is not right. From such model it follows that electron mean free path must be of the order of the lattice constant, what is about one or two orders smaller than the real electron mean free path.

The randomly moving electrons in geometric ideal lattice produce the local fluctuation of the charge due to escape some of electrons to distance of the free path. Such deviation from the ideal periodicity of the potential energy is named by "electronic defect" [13], it is caused by not completely screened native material ion by electrons (in future we will use the term "electronic defect" for description of such defect). There we want to point that thermal vibrations of the lattice play other role, than it has been described in $[1-8]$. With temperature increase there at the Fermi surface are excited the electrons, which can be scattered and their mean free path is a many orders higher than the lattice constant. The density of such electrons is equal to $n_{\text {eff }}=g\left(E_{\mathrm{F}}\right) k T$. At the same time there are produced the same number of distortion spots $N_{\text {eff }}$ from the ideal periodicity of the potential energy distribution due to appearance native material ions not completely screened by electrons (electronic defects): $N_{\text {eff }}=n_{\text {eff }}=g\left(E_{\mathrm{F}}\right) k T$. These electronic defects cause the scattering of the free electrons on the Fermi surface, and produce the random moving of free electrons.

Then the mean free path $l_{\mathrm{F}}$ and average relaxation time $\tau_{\mathrm{F}}$ of scattered electrons for metals can be described as

$l_{\mathrm{F}}=v_{\mathrm{F}} \tau_{\mathrm{F}}=1 /\left(\sigma_{\text {eff }} N_{\text {eff }}\right)=1 /\left[\sigma_{\text {eff }} g\left(E_{\mathrm{F}}\right) k T\right]$,

$\tau_{\mathrm{F}}=1 /\left(\sigma_{\text {eff }} N_{\text {eff }} v_{\mathrm{F}}\right)=1 /\left(\sigma_{\text {eff }} g\left(E_{\mathrm{F}}\right) \nu_{\mathrm{F}} k T\right)$,

where $\sigma_{\text {eff }}$ is the effective electron scattering cross-section due to electron scattering by electronic defects.

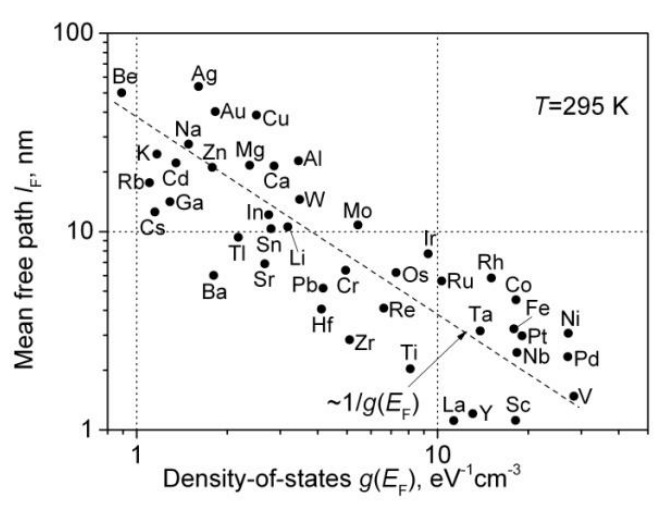

Fig. 2. Mean free path $l_{\mathrm{F}}$ (Eq. 16) distribution on DOS $g\left(E_{\mathrm{F}}\right)$ for elemental metals at $T=295 \mathrm{~K}\left(\mathrm{DOS} g\left(E_{\mathrm{F}}\right)\right.$ are taken from [13])

The mean free path of RM electrons distribution on DOS for different elemental metals at $T=295 \mathrm{~K}$ is presented in Fig. 2. Considering that $n_{\mathrm{eff}}=g\left(E_{\mathrm{F}}\right) k T$ at a given temperature linearly depends on DOS $g\left(E_{\mathrm{F}}\right)$, it means that analogical patterns will depend on $n_{\text {eff. }}$.

From Eq. 16 it follows that electron mean free path dependence on temperature above the Debye's temperature $\Theta$ is completely determined by effective density of electronic defects $N_{\text {eff. }}$. From Eq. 16 also follows that effective electron scattering cross-section $\sigma_{\text {eff }}$ doesn't depend on temperature above the Debye's temperature $\Theta$. So, the statement that scattering cross-section at this temperature range is proportional to temperature $[1-8]$ is not right. The Eq. 16 directly shows that electron mean free path is in inverse proportion not only to temperature, but also to DOS at the Fermi surface.

In paper [16], it was shown that electron mean free path in the linear resistivity dependence on temperature range can be expressed by the diffusion coefficient for RM electrons of elemental metals as

$l_{\mathrm{F}}(T)=1.39\left(D\left(T_{0}\right)\right)^{2 / 3}\left(T_{0} / T\right)$,

where $l_{\mathrm{F}}$ is in $\mathrm{nm}$, and $D\left(T_{0}\right)$ is in $\mathrm{cm}^{2} / \mathrm{s}$ at $T_{0}=295 \mathrm{~K}$. The calculation results obtained from Eq. 18 well agree to the data published in paper [16], and data estimated from the dimension effect [17]. The Eq. 18 at $T>\Theta$ can be performed as

$l_{\mathrm{F}}(T)=21.9(\beta)^{2 / 3}\left(T_{0} / T\right)$,

where $l_{\mathrm{F}}$ is in $\mathrm{nm}$, non-dimensional parameter $\beta=\sigma_{\mathrm{rel}} / g_{\mathrm{rel}}\left(E_{\mathrm{F}}\right)$ is estimated at $T=T_{0}=295 \mathrm{~K}$, where the 
relative conductivity $\sigma_{\text {rel }}=\sigma / \sigma_{0}=\sigma / 10^{5}: \sigma=\sigma(295 \mathrm{~K})$ and $\sigma_{0}=10^{5}$ are in units $\Omega^{-1} \mathrm{~cm}^{-1}$; the relative DOS $g_{\text {rel }}\left(E_{\mathrm{F}}\right)=g\left(E_{\mathrm{F}}\right) / g_{0}\left(E_{\mathrm{F}}\right)=g\left(E_{\mathrm{F}}\right) / 10^{22}: g\left(E_{\mathrm{F}}\right)$ and $g_{0}\left(E_{\mathrm{F}}\right)=10^{22}$ are in units $\mathrm{eV}^{-1} \mathrm{~cm}^{-3}$. The data for calculation of parameter $\beta$ are taken from [13].

In similar way, the average electron velocity $v_{\mathrm{F}}$ at Fermi surface for elemental metals can be expressed as

$v_{\mathrm{F}}=8.58(\beta)^{1 / 3}$,

here $v_{\mathrm{F}}$ is in units $10^{5} \mathrm{~m} / \mathrm{s}$ and does not depend on temperature.

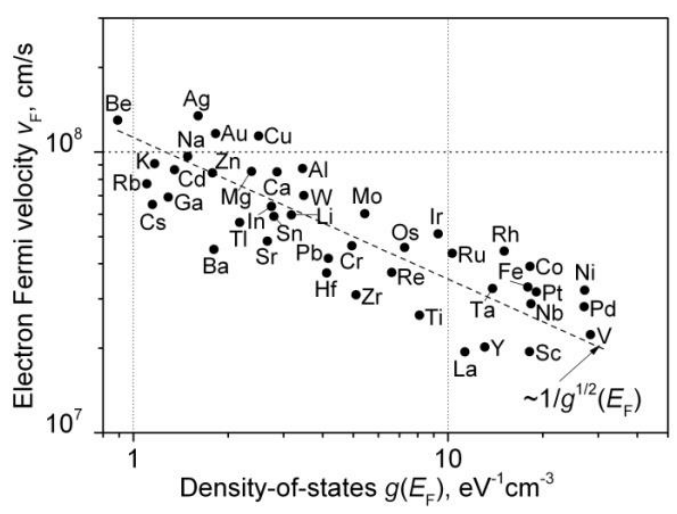

Fig. 3. Distribution of the electron Fermi velocity $v_{\mathrm{F}}$ (Eq. 20) on the DOS $g\left(E_{\mathrm{F}}\right)$ at the Fermi surface for elemental metals (DOS $g\left(E_{\mathrm{F}}\right)$ are taken from [13])

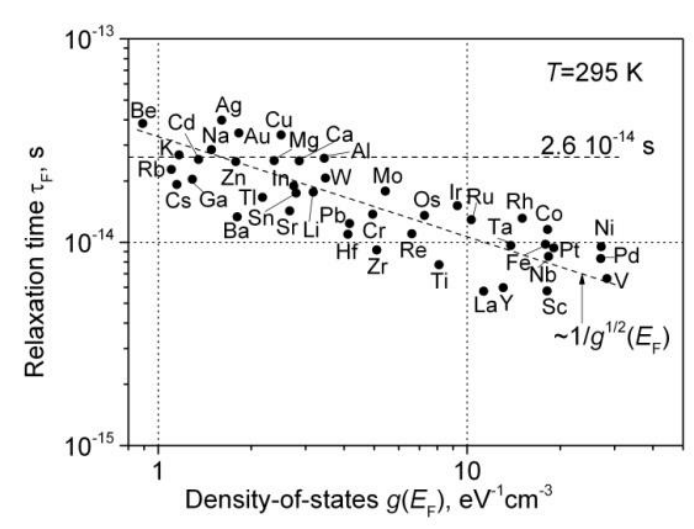

Fig. 4. Electron average relaxation time $\tau$ F (Eq. 21) distribution on DOS $\mathrm{g}\left(E_{\mathrm{F}}\right)$ for elemental metals at $\mathrm{T}=295 \mathrm{~K}\left(\mathrm{DOS} g\left(E_{\mathrm{F}}\right)\right.$ are taken from [13]; the value $2.6 \times 10^{-14} \mathrm{~s}$ is according to [19])

The electron average relaxation time $\tau_{\mathrm{F}}$ at $T>\Theta$ can be described as

$\tau_{\mathrm{F}}=2.54(\beta)^{1 / 3}\left(T_{0} / T\right)$,

where $\tau_{\mathrm{F}}$ is in units $10^{-14} \mathrm{~s}$. The distribution of the electron Fermi velocity on DOS for different metals is presented in Fig. 3. From this figure it is seen that electron Fermi velocity on average decreases with DOS increasing as $v_{\mathrm{F}} \sim 1 / g^{1 / 2}\left(E_{\mathrm{F}}\right)$.

In Fig. 4, there is shown the distribution of average electron relaxation time on the DOS at Fermi surface for elemental metals. There must be pointed that statement that average relaxation time for all metals in the range of the linear resistivity dependence on temperature can be expressed as $\tau_{F} \cong \hbar / k T$ [19] is not right, because the average relaxation times for different metals at room temperature are in range $(0.6-4) 10^{-14} \mathrm{~s}$ (Fig. 4), while this value under [19] at $T=295 \mathrm{~K}$ must be about $2.6 \times 10^{-14} \mathrm{~s}$ (horizontal dash line in Fig. 4.

The effective electron scattering cross-sections data calculated using Eq. 16 and Eq. 19 are presented in Fig. 5. The effective electron scattering cross-sections are distributed in the range $(0.40-3.6) 10^{-15} \mathrm{~cm}^{2}$, and they do not depend on temperature for that temperature range where the resistivity is proportional to temperature $T$.

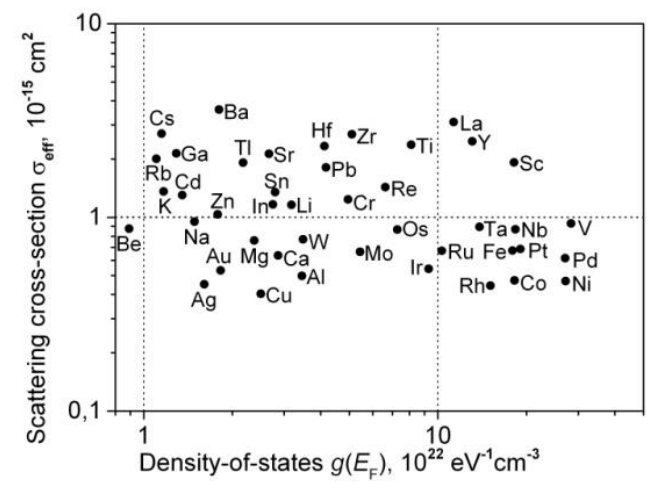

Fig. 5. The effective scattering cross-section (calculated from Eq. 16 and Eq. 19) distribution on DOS for elemental metals above the Debye's temperature (DOS $g\left(E_{\mathrm{F}}\right)$ are taken from [13]; $\sigma_{\text {eff }}$ is calculated by Eq. 16 at $T=295 \mathrm{~K}$ )

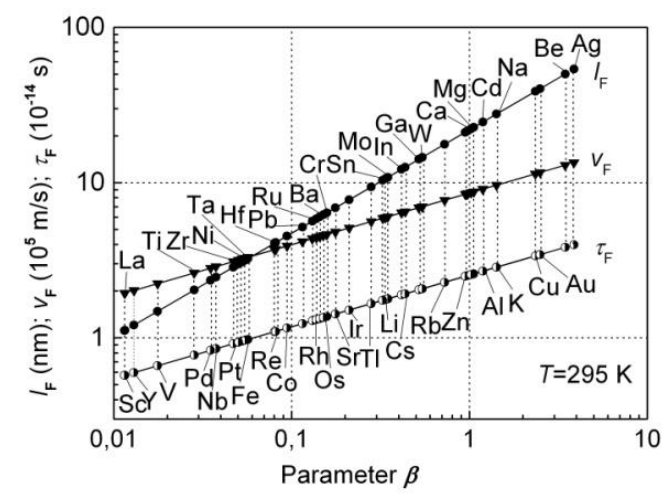

Fig. 6. Relationships of the mean free path $l_{F}$, average velocity $v_{\mathrm{F}}$, and average relaxation time $\tau_{\mathrm{F}}$ of randomly moving charge carriers dependences on parameter $\beta$ for different metals at $T=295 \mathrm{~K}$. The data dots for the same metals are connected with vertical dash lines.

The effective electron scattering cross-section for every metal has a particular value, which depends on the electronic defect structure. The results presented in Fig. 2 -Fig. 5 show that RM electrons basic parameter such as mean free path, Fermi velocity, relaxation time, and effective scattering cross-section values are scattered, and have some tendency of changes with DOS at Fermi energy.

From obtained results follows that the resistivity linear dependence on temperature above the Debye temperature $\Theta$ is caused by electronic defect density $N_{\text {eff }}$ dependence on 
temperature with constant scattering cross-section. In the case of electron-phonon scattering the cross-section must increase with temperature increasing as $T$, and it would originate the resistivity dependence on temperature as $T^{2}$ in the range of temperature $T>\Theta$, but it does not take place.

From Eq. 19-Eq. 21 it is possible to design the following important pattern (Fig. 6) where there are shown the relationships of the mean free path, average velocity, and the average relaxation time of the RM electrons with parameter $\beta$ for elemental metals at room temperature. The results presented in Fig. 6 integrate the main transport properties of randomly moving electrons in metals.

\subsection{Influence of phonons to resistivity of metals below the Debye's temperature}

In Fig. 7 there are presented the measurement results of electrical resistivity dependences on temperature for gold and tungsten [20-22]. What effects cause such steep resistivity decreasing with temperature in temperature range below the Debye's temperature $\Theta$ ?

The independent resistivity part on temperature below $10 \mathrm{~K}$ is the residual electrical resistivity due to scattering of electrons from chemical and structural imperfections in the lattice of the investigated samples.

The intensity of the scattering of electrons by electronic defects depends on the exchange of thermal energies between phonon and electron. Accounting that electron scattering by electronic defect is inelastic, and that electron energy fluctuations cause the excitation and annihilation of phonons, then the ratio of the exchange of the thermal energies between phonon and RM electron can be described as $[4,16]$ :

$$
\frac{E_{p h 1}}{E_{e l 1}}=\frac{3 k T}{1.64 k T} \eta(T / \Theta) \approx 1.83 \eta(T / \Theta)
$$

where

$$
\eta(T / \Theta)=\left(\frac{T}{\Theta}\right)^{4 \Theta / T} \int_{0}^{4 x^{5}} \frac{\left(e^{x}-1\right)\left(1-e^{-x}\right)}{\mathrm{d} x}
$$

is the phonon mediation factor for electron scattering on electronic defects, and $\Theta$ is the Debye's temperature?

Then the metal resistivity in the overall temperature range can be described as

$$
\rho_{\text {res }}=\rho_{0}+\rho\left(T_{0}\right) \cdot\left(T / T_{0}\right) \cdot \eta(T / \Theta),
$$

where $\rho_{0}$ is the residual electrical resistivity due to scattering of electrons from imperfections of the lattice structure, $T_{0}$ is the reference temperature for the linear range of the resistivity dependence on temperature (here for calculation we take $T_{0}=300 \mathrm{~K}$ ). So, the phonon mediated resultant electron scattering cross-section $\sigma_{\text {res }}$ can be described as

$\sigma_{\text {res }}=\sigma_{\text {eff }} \eta(T / \Theta)$,

where the effective electron scattering cross-section $\sigma_{\mathrm{eff}}$ is independent on $T$, and it accounts the constant multiplier 1.83 from Eq. 22. The resultant average relaxation time $\tau_{\text {res }}$ can be described as
$1 / \tau_{\text {res }}=\left(1 / \tau_{\text {eff }}\right)+\left(1 / \tau_{\text {imp }}\right)$,

where $\quad \tau_{\text {eff }}=1 /\left(\sigma_{\text {res }} N_{\text {eff }} v_{\mathrm{F}}\right)=1 /\left(\sigma_{\text {res }} g\left(E_{\mathrm{F}}\right) v_{\mathrm{F}} k T\right)$, and $\tau_{\text {imp }}=1 /\left(\sigma_{\text {imp }} N_{\text {imp }} v_{\mathrm{F}}\right) ; \sigma_{\text {imp }}$ is the average scattering crosssection of the impurity (imperfection) defect density $N_{\text {imp }}$.

The calculation results for $\mathrm{Au}$ and $\mathrm{W}$ electrical resistivity dependence on temperature by using Eq. $22-$ Eq. 25 are presented in Fig. 7 by solid lines. The Debye's temperature $\Theta$ has been chosen to the best agreement between calculation data and experimental dots. Here the calculations have been performed by using constant quantity $\Theta: \Theta_{\mathrm{Au}}=185 \mathrm{~K}$ and $\Theta_{\mathrm{W}}=350 \mathrm{~K}$.

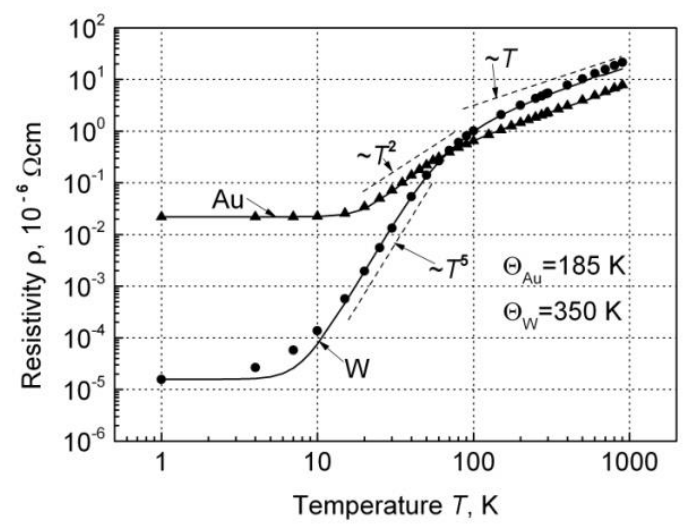

Fig. 7. The electrical resistivity dependence on temperature for gold and tungsten (dots are the experimental results [20-22], the solid lines are the calculations data by using Eq. 24

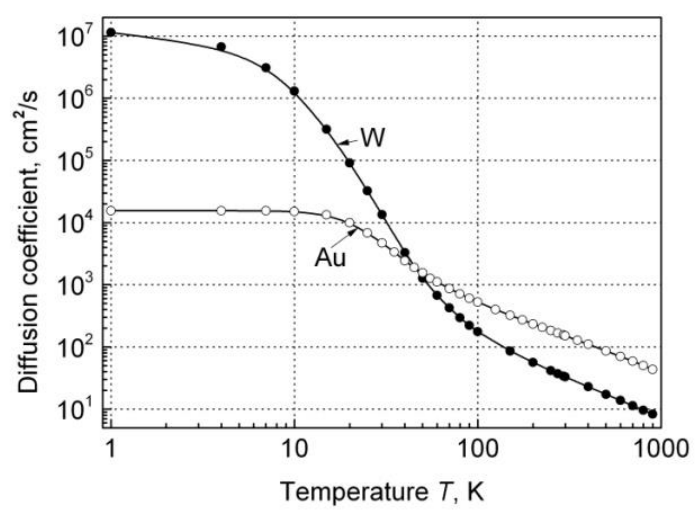

Fig. 8. Electron diffusion coefficient $D$ dependence on temperature for $\mathrm{Au}$ and $\mathrm{W}$ calculated by using relation $D(T)=1 /\left[q^{2} \rho(T) g\left(E_{\mathrm{F}}\right)\right]$

Applying the following sequence:

$$
D(T)=\frac{1}{q^{2} g\left(E_{\mathrm{F}}\right) \rho(T)} \Rightarrow l_{\mathrm{F}}(T)=\frac{3 D(T)}{v_{\mathrm{F}}} \Rightarrow \tau_{\mathrm{F}}(T)=\frac{l_{F}(T)}{v_{\mathrm{F}}}
$$

and using the measurement data of electrical resistivity for gold and tungsten [20-22] (Fig. 7) it is easy to find their basic kinetic characteristics in temperature range from $1 \mathrm{~K}$ to $900 \mathrm{~K}$. In Fig. 8 there is presented the electron diffusion coefficient $D(T)$ of the RM electrons dependence on temperature for $\mathrm{Au}$ and $\mathrm{W}$. 
The comparison of the mean free path $l_{\text {mean }}(T)$ and the resultant electron relaxation time $\tau_{\text {res }}(\mathrm{T})$ of the RM electrons dependences on temperature for these metals in temperature range from $1 \mathrm{~K}$ to $900 \mathrm{~K}$ are presented in Fig. 9 and Fig. 10, respectively. For the most of metals the atom cross-section is in the range $(0.5-1.2) \cdot 10^{-15} \mathrm{~cm}^{2}$ [3] (here for estimation of impurity density $N_{\mathrm{imp}}$ we take $\left.\sigma_{\mathrm{imp}} \approx 0.7 \cdot 10^{-15} \mathrm{~cm}^{2}\right)$.

Thus, considering that density-of-states $g\left(E_{\mathrm{F}}\right)$, and velocity of electrons at Fermi surface $v_{\mathrm{F}}$ don't depend on temperature, it is easy entirely from resistivity (conductivity) data to find the basic electron transport characteristics of metals. Of course, it does not include the temperature range where particular metal is in superconducting state.

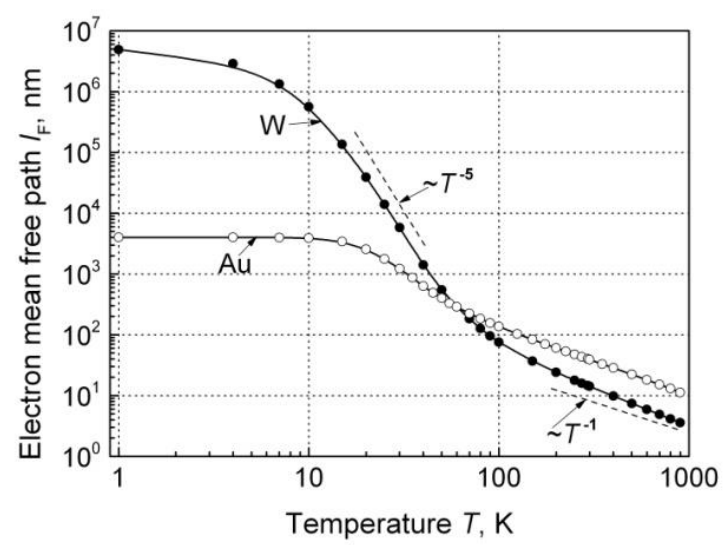

Fig. 9. Electron mean free path $l_{\mathrm{F}}$ dependence on temperature for $\mathrm{Au}$ and $\mathrm{W}$ by using relation $l_{\mathrm{F}}(T)=3 D(T) / \nu_{\mathrm{F}}$

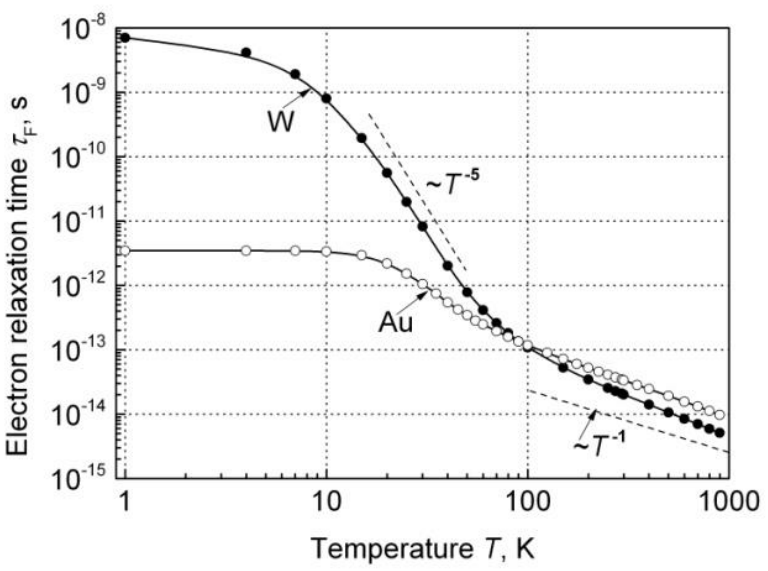

Fig. 10. The resultant electron relaxation time dependence on temperature for $\mathrm{Au}$ and $\mathrm{W}$ by using relation $\tau_{\text {res }}=l_{\text {mean }} / \nu_{\mathrm{F}}$

It is interesting to note that at cryogenic temperature range the conductivity and the electron mean free path for tungsten is about three orders higher than that in gold. It is because the residual neutral impurity density in gold at this temperature range is about $0.01 \%$, while in tungsten it reaches only $3 \cdot 10^{-6} \%$.

\section{CONCLUSIONS}

In this work on the base of randomly moving (RM) electrons it is shown that the resistivity dependence on temperature for elemental metals mainly is due to RM electrons scattering by electronic defects, accounting the exchange thermal energies between phonon and RM electron. There are presented the general relationships for estimation of the average diffusion coefficient, the average velocity, mean free length and average relaxation time of $\mathrm{RM}$ electrons at the Fermi surface in very wide temperature range. The effective electron scattering cross-sections for all elemental metals also are estimated, and it is shown that the effective electron scattering cross-section does not depend on temperature above the Debye temperature.

There also is presented the simple method for determination of the metal basic kinetic characteristic dependence on temperature only from the resistivity dependence on temperature. It is at first time demonstrated for $\mathrm{Au}$ and $\mathrm{W}$ the temperature dependences of the mean free path, average diffusion coefficient, average relaxation time of RM electrons from $1 \mathrm{~K}$ to $900 \mathrm{~K}$.

\section{REFERENCES}

1. Sondheimer, E.H. The Mean Free Path of Electrons in Metals Advances in Physics 50 (6) 2001: pp. 499-537. https://dx.doi.org/10.1080/00018730110102187

2. Kaveh, M., Wiser, N. Electron-electron Scattering in Conducting Materials Advances in Physics 33 (4) 1984: pp. 257-372. https: //dx.doi.org/10.1080/00018738400101671

3. Ashcroft, N.W., Mermin, N.D. Solid State Physics (ch. 2). HRW International Edition, New York, Chicago, 1976: pp. $01-56$.

4. Ziman, J.M. Principles of the Theory of Solids. Cambridge University Press, Cambridge, Online 2013: pp. 211-254. https://doi.org/10.1017/CBO9781139644075

5. Abrikosov, A.A. Principles of the Theory of Metals. Dover publication, USA, 2017: pp. 33-45.

6. Ziman, J.M. Electrons and Phonons. The Theory of Transport Phenomena in Solids, Oxford Classic Texts in thePhysical Sciences, Oxford University Press, Oxford, 2001: pp. $334-418$.

7. Lundstrom, M. Fundamentals of Carrier Transport. Cambridge Books Online, Cambridge University Press, Cambridge, 2014: pp. 158-211. https://dx.doi.org/10.1017/CBO9780511618611.007

8. Rossiter, P.L. The Electrical Resistivity of Metals and Alloys. Cambridge Books Online, Cambridge University Press, Cambridge, 2014: pp. $107-136$. https://dx.doi.org/10.1017/CBO9780511618600289.005

9. Palenskis, V. Drift Mobility, Diffusion Coefficient of Randomly Moving Charge Carriers in Metals and Other Materials with Degenerate Electron Gas World Journal of Condensed Matter Physics 3 (3) 2013: pp. 73-81. https://dx.doi:10.4236/wjcmp.2013.31013

10. Palenskis, V. The Effective Density of Randomly Moving Electrons and Related Characteristics of Materials with Degenerate Electron Gas AIP (American Institute of Physics) Advances 4 (4) 2014: pp. 047119-1(9). https://dx.doi.org/10.1063/1.4871757

11. Cracknell, A.P., Wong, K.C. The Fermi Surfaces: Its Concept, Determination, and Use in the Physics of Metals. Clarendon Press, Oxford, 1973: pp. 270-360. https://trove.nla.gov.au/version/26010077 
12. Seeger, K. Semiconductor Physics: An Introduction. Springer, Berlin, 2004: pp. 46-152.

13. Kittel, Ch. Introduction to Solid States Solids. John Wiley and Sons, Inc., New York, London, 1976: pp. 141-172.

14. Dugdale, J.S. The Electrical Properties of Disordered Metals. Cambridge University Press, Cambridge, 2010: pp. 20-47 https://dx.doi.org/10.10.17/CBO09780511629020

15. Palenskis, V. Transport of Electrons in Donor-doped Silicon at Any Degree of Degeneracy of Electron Gas World Journal of Condensed Matter Physics 4 (3) 2014: pp. 123-133. https://dx.doi:10.4236/wjcmp.2014.43017

16. Palenskis, V., Žitkevičius, E. Phonon Mediated Electronelectron Scattering in Metals World Journal of Condensed Matter Physics 8 (3) 2018: pp. 115-129. https://dx.doi:10.4236/wjcmp.2018.83008

17. Gall, D. Electron Mean Free Path in Elemental Metals Journal of Applied Physics 119 2016: pp. 085101 -1(5). https://doi.org/10.1063/1.4942216
18. Chopra, K.L. Thin Film Phenomena. R. E. Krieger Publication Company, Science, Malabar, 1969 (Translation to Russian, Mir: Moscow, 1972: pp. $142-172$ ).

19. Devillers, M.A.C. Lifetime of Electrons in Metals at Room Temperature Solid State Communications 49 1984: pp. 1019-1022. https://doi.org/10.1016/0038-1098(84)90413-7

20. http://www.knowledgedoor.com/2/elements_handbook/elect rical_resistivity.html

21. Matula, R.A. Electrical Resistivity of Copper, Gold, Palladium, and Silver Journal of Physical and Chemical Reference Data 8 (4) 1979: pp. 1147 - 1298. https://doi:10.10.64/1.555614.19

22. Desai, P.D., Chu, T.K., James, H.M., Ho, C.Y. Electrical Resistivity of Selected Elements Journal of Physical and Chemical Reference Data 13 (4) 1984: pp. 1069-1096. 